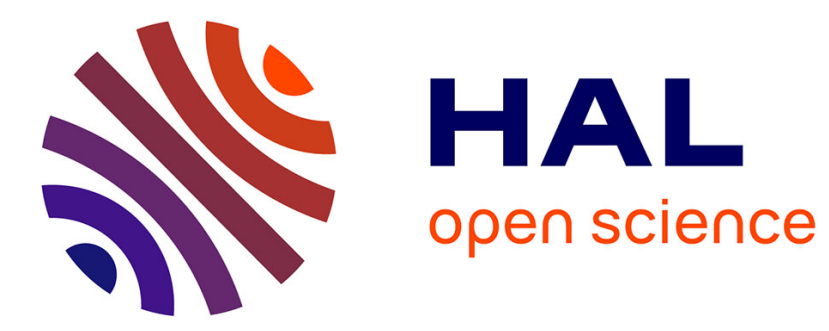

\title{
Critical subsystems in time interval models Philippe Declerck
}

\section{To cite this version:}

Philippe Declerck. Critical subsystems in time interval models. Discrete Event Dynamic Systems, 2020, 10.1007/s10626-020-00322-0 . hal-03148670

\section{HAL Id: hal-03148670 \\ https://univ-angers.hal.science/hal-03148670}

Submitted on 22 Feb 2021

HAL is a multi-disciplinary open access archive for the deposit and dissemination of scientific research documents, whether they are published or not. The documents may come from teaching and research institutions in France or abroad, or from public or private research centers.
L'archive ouverte pluridisciplinaire HAL, est destinée au dépôt et à la diffusion de documents scientifiques de niveau recherche, publiés ou non, émanant des établissements d'enseignement et de recherche français ou étrangers, des laboratoires publics ou privés. 


\title{
Critical subsystems in Time Interval Models Application to a baking process
}

\section{P. Declerck}

Received: date / Accepted: date

\begin{abstract}
The aim of this paper is the generation of the min-critical and max-critical subsystems which determine the optimal cycle times. Considering a Time Interval Model which can describe Timed Event Graphs and P-time Event Graphs completely, each critical subsystem depends on the lower and upper bounds of the time durations. The proposed approach which is based on linear programming makes a classification of the relations which describe the system. The application to a baking process in a plant bakery shows that the min-critical and max-critical subsystems are not limited to the critical circuits of the Event Graph.
\end{abstract}

Keywords Time Interval Model · P-time Event Graph · cycle time · critical · linear programming

\section{Introduction}

Context and aim. In the field of discrete event systems, the study of time is a fundamental topic and the dater description is an efficient tool which can describe complex time phenomena as synchronizations with different specific semantics, token deaths and additional time constraints. The studies [4] [3] consider a general model, named Time Interval Model below, which is described by an algebraic model $A . x \leq b$ and can cover a large number of models such as Timed Event Graphs, P-time Event Graphs and P-time Event Graphs with Affine-Interdependent Residence Durations [4] [3]. As the number of rows of $A$ is twice the number of places and, each row of matrix $A$ can contain more than two entries which can be different from 1 and -1 in general (despite

P. Declerck

LISA/LARIS, University of Angers, 62 avenue Notre-Dame du Lac, 49000 Angers, France Tel.: +33241226560

Fax: +33241 226561

E-mail: philippe.declerck@univ-angers.fr 
that all arc weights of the Event Graphs are equal to 1), matrix $A$ is not the incidence matrix of the event graph. So, classical techniques determining the circuits (or equivalently P-invariants) and computing the maximum of the classical ratios (defined by the sum of temporizations to the sum of the number of the initial tokens, for each elementary circuit) cannot directly be used, and an adaptation must be made. In addition, a simple search of any circuit faces a combinatorial explosion of the number of circuits. Therefore, most general tools such as the linear programming must be exploited. Based on a Farkas' Lemma and Stiemke's theorem, the papers [3] [4] analyze the existence of the extremum cycle times and propose techniques allowing the computation of the optimal trajectories.

This paper was motivated by the problem of finding the bottleneck subnets which determine the minimum and maximum production rates of the production system. Applications can be found in the food industry and the transportation networks as railway nets or road networks: Typically, public works in a transportation network can reduce the general speed of the entire system for some specific localizations, but, this consequence is not systematic as the effect can be null for some other localizations. For the management of these works, it is important to determine these critical localizations (resp. non-critical localizations) where a parameter variation as a longer journey time of a particular train implies (resp. does not imply) a degradation of the best production rate of the complete process. The reliability of these critical subsystems is also crucial to ensure an adequate production rate. Another application is the vulnerability analysis of a critical infrastructure, as the crude-oil distribution network of Saudi Arabia, whose aim is to identify the critical parts of the network which should be protected against coordinated attacks [2].

In this paper, we analyze the relations of the Time Interval Model which can describe the tasks expressed by the places of Timed Event Graphs or Ptime Event Graphs. We focus on the determination of the critical relations which determine the lower and upper bounds of the optimal cycle time and are decisive for the optimal cycle times. These critical relations are defined as follows: a relation is said critical if there is a small variation of a time duration which produces a degradation of the optimal cycle time of the complete system and reduces its optimality. In the paper, we show that a variation of a time duration for a unique critical relation can lead to unexpected variations of the cycle times such as an increase of the minimum cycle time (which is a slow-down of the entire system if we desire the fastest rate) or a decrease of the maximum cycle time (which is an acceleration of the whole system if we desire the slowest rate).

The paper is organized as follows: firstly, some related works are presented below. In Section 2, we describe the algebraic model of P-time Event Graphs which allows to illustrate the approach. Section 3 presents some preliminary results in subsection 3.1, gives the main objective in subsection 3.2 and describes the techniques to determine the critical relations in subsection 3.3. Finally, the approach is illustrated by a baking process in a plant bakery in Section 3.4. 
Related works. By lack of place, we only give some related works. Time can be associated with places, transitions and arcs of the Petri nets. Many studies [5] consider Timed Event Graphs where the time durations of the task is associated to places. The computation of the cycle time can be made by building a transfer function of formal power series in two variables $\gamma$ and $\delta[1]$, but this technique faces numerical difficulties as a non-polynomial time complexity and the existence of a transient period which can lead to an extremely long transfer function even for small systems. A generalization is the class of P-time Event Graphs where a time interval is associated with the places. In the same spirit, the paper [7] considers a model named Negative Event Graph which corresponds to a P-time Event Graph; The earliest and latest feasible steady firing schedules for each of the minimum and maximum cycle times are examined and the liveness is discussed. The article [8] analyzes a linear precedence constraints graph where the labels of the arcs bring a complex form of numbering of the starting times; A difference with P-time Event Graphs is that the processing times present a minimum delay but not a maximum time duration.

Different from the class of the P-time Petri nets, another class of models is the Time Petri nets that assign time or a time interval to each transition enabled by the marking. Focusing essentially on the determination of some parameters as the average marking but not on the optimal trajectories, the quantitative analysis of these models basically consists in applying enumerative techniques based on the construction of graphs as the graph of state classes or the reachability graph which suffer the well-known state space explosion problem even in the case of bounded Petri nets. To tackle this drawback, several methods avoid the construction of the whole state space. In [9], the computation of the throughput bounds is made with linear programming. To compute more accurate bounds in Stochastic Event Graphs, the strategy in [10] is to add a cycle potentially more restrictive at each iteration. The different basic quantities computed in [2] depend on an experiment interval whose value can modify the results. Another point is that these studies have a "performance monotonicity property", that is a local pessimistic transformation, such as incrementing the average firing times, leads to a slower transformed net system. This property implies that these papers can use the extreme points of the transition interval firing times for the computation of their performance bounds contrary to this proposed paper where the considered models do not presents this property: in general, each optimal bound of the production rate depends on the lower and upper bounds of the places together.

\section{Petri nets and algebraic model}

In the paper, "resp." is an abbreviation of "respectively". The notation $|E|$ stands for the cardinality of the set $E$ while the notation $A_{i, \text {. corresponds to }}$ row $i$ of matrix $A$. The transpose of the matrix $A$ is denoted $A^{t}$. We consider the 'dater' representation well-known in $(\max ,+)$ algebra [1]: each variable 
$x_{i}(k)$ over $\mathbb{R}$ with $k \in \mathbb{N}$ represents the date of the $k^{t h}$ event associated with $x_{i}$. A brief description of the Petri nets and P-time Event Graphs is now presented. A Petri net is a pair $\left(G R, \mathbf{M}^{0}\right)$, where $G R=(V, A R)$ is a bipartite graph defined as follows: the set $V$ is a finite number of nodes which are partitioned into disjoint sets of places $P$ and transitions $T R$; the set $A R$ consists of pairs of the form $\left(p_{i}, x_{j}\right)$ and $\left(x_{j}, p_{i}\right)$ with $p_{i} \in P$ and $x_{j} \in T R$. The initial marking $\mathbf{M}^{0}$ is a vector of dimension $|P|$ whose elements denote the number of initial tokens in the respective places and $\mathbf{M}_{i}^{0}$ is the initial marking of the place $p_{i} \in P$. The set ${ }^{\bullet} p_{i}$ (resp., $p_{i}^{\bullet}$ ) is the set of input (resp., output) transitions of $p_{i} \in P$. The set ${ }^{\bullet} x_{i}$ (resp., $x_{i}^{\bullet}$ ) is the set of input (resp., output) places of the transition $x_{i} \in T R$. For a Petri net with $|P|$ places and $|T R|$ transitions, the incidence matrix $W=\left[W_{i j}\right]$ is a $|P| \times|T R|$ matrix of integers and its entry is given by $W_{i j}=W_{i j}^{+}-W_{i j}^{-}$where $W_{i j}^{+}$is the weight of the arc from transition $j$ to place $i$, and $W_{i j}^{-}$is the weight of the arc from place $i$ to transition $j$. In a Petri net, a firing sequence from a marking $\mathbf{M}$, implies a sequence of successive markings. The characteristic vector $s$ of a firing sequence $S$ is such that each component of $s$ is a natural number corresponding to the number of firings of the corresponding transition. A marking $\mathbf{M}$ reached from an initial marking $\mathbf{M}^{0}$ by the firing of a sequence $S$, can be calculated by the fundamental relation: $\mathbf{M}=\mathbf{M}^{0}+W . s$ where $W . s$ is the usual matrix product of $W$ by vector $s . \mathrm{A}$ Petri net is called an Event Graph (also named Marked Graph) if each place has exactly one input and one output transition: formally, $\left|{ }^{\bullet} p\right|=\left|p^{\bullet}\right|=1$ $(\forall p \in P)$. All arc weights of the event graphs considered below are unitary. In Petri Nets (PNs), time can be associated with places, transitions or arcs. A Timed Event Graph is a triple $\left(G R, \mathbf{M}^{0}, f\right)$ where $\left(G R, \mathbf{M}^{0}\right)$ is an Event Graph and $f: P \rightarrow \mathbb{R}^{+}$is the mapping that assigns a holding time to each place. This duration represents the time that a token must spend in the place before it can play its role in the enabling of the downstream transition. The transition firings are supposed to be instantaneous. For Time PNs, temporal intervals can be associated with places or transitions but the corresponding subclasses (P-Time PNs and T-Time PNs ) are fundamentally different. In T-Time PNs, a temporal interval is associated with each transition enabled by the marking while a temporal interval of availability is associated with each token which enters a place in P-Time PNs. In Time Stream PNs, temporal intervals are associated with arcs outgoing from places and the firing interval of transitions is defined by different semantics [5][11]. We now define P-time Event Graphs.

P-time Petri nets allow the modeling of discrete event systems with time constraints on tokens. We associate a temporal interval defined in $\mathbb{R}^{+} \times\left(\mathbb{R}^{+} \cup\right.$ $\{+\infty\})$ with each place: each place $p_{l} \in P$ is associated with an interval $\left[T_{l}^{-}, T_{l}^{+}\right]$, where $T_{l}^{-}$is the lower bound and $T_{l}^{+}$is the upper bound. A P-time Event Graph is a triple $\left(G R, \mathbf{M}^{0}, f\right)$ where $\left(G R, \mathbf{M}^{0}\right)$ is an Event Graph, and the mapping $f: P \rightarrow \mathbb{R}^{+} \times\left(\mathbb{R}^{+} \cup\{+\infty\}\right)$ associates with each place $p_{l}$ an interval $\left[T_{l}^{-}, T_{l}^{+}\right]$with $0 \leq T_{l}^{-} \leq T_{l}^{+}$. The interval $\left[T_{l}^{-}, T_{l}^{+}\right]$is the static interval of duration time of a token in place $p_{l}$. The token must stay in this place during the minimum residence duration $T_{l}^{-}$. Before this duration, the 
token is in a state of unavailability for firing the outgoing transition. The value $T_{l}^{+}$is a maximum residence duration after which the token must leave place $p_{l}$ (and can contribute to the enabling of the downstream transition). If the token does not leave the place before $T_{l}^{+}$, it dies. So, the token is available to fire the outgoing transition in the time interval $\left[T_{l}^{-}, T_{l}^{+}\right]$.

Each considered event is the firing of transition $x_{i} \in T R$ and for the sake of simplicity the relevant date is usually denoted $x_{i}(k) \in \mathbb{R}$ with $k \in \mathbb{N}$ where $x_{i}(0)$ is the first firing date of $x_{i}$. Let $m \in \mathbb{N}$ be the maximum number of the initial tokens: $m=\max \left\{\mathbf{M}_{l}^{0} \mid l \in[1,|P|]\right\}$. If we assume a FIFO functioning of the places which guarantees that the tokens do not overtake one another and the absence of token deaths, a correct numbering of the events can be carried out. A P-time Event Graph can be described by the following system

$$
T_{l}^{-} \leq x_{p_{l}}(k)-x \cdot p_{l}\left(k-\mathbf{M}_{l}^{0}\right) \leq T_{l}^{+}
$$

for each place $p_{l} \in P$ where each place $p_{l}$ links one upstream transition $\left\{x_{j}\right\}=$ - $p_{l}$ and one downstream transition $\left\{x_{i}\right\}=p_{l}^{\bullet}$. The difference $x_{p_{l}}(k)-x_{\bullet} p_{l}(k-$ $\left.\mathbf{M}_{l}^{0}\right)$ is the time duration of the presence of a token generated at $x \cdot p_{l}\left(k-\mathbf{M}_{l}^{0}\right)$ and deleted just after $x_{p_{i}}(k)$.

Without reduction of generality, we can suppose that $m=1$, that is $\mathbf{M}_{l}^{0} \in$ $\{0,1\}$ which can be obtained by the building technique given in appendix of [3]. Therefore, system (1) can be rewritten under the following algebraic model (2) defined over $\mathbb{R}$ which is named Time Interval model (TIM). Each row of (2) corresponds to a pair $\left(p_{l}, T_{l}^{-}\right)$for $i \in\{1, \ldots,|P|\}$ or $\left(p_{i-|P|}, T_{i-|P|}^{+}\right)$ for $i \in\{|P|+1, \ldots, 2 .|P|\}$. So,

$$
\left(\begin{array}{l}
G^{-} \\
G^{+}
\end{array}\right) \cdot\left(\begin{array}{l}
x(k) \\
x(k+1)
\end{array}\right) \leq\left(\begin{array}{c}
-T^{-} \\
T^{+}
\end{array}\right)
$$

for $k \in \mathbb{N}$ where: $n=|T R|$ and $q=|P|$ are natural numbers; $x(k)$ and $x(k+1) \in \mathbb{R}^{n} ; T^{-}$and $T^{+} \in(\mathbb{R} \cup\{-\infty,+\infty\})^{q} ; G^{-}=\left(G_{1}^{-} G_{0}^{-}\right)$and $G^{+}=$ $\left(G_{1}^{+} G_{0}^{+}\right) \in \mathbb{R}^{q \times 2 . n}$.

Matrices $G^{-}$and $G^{+}$express time connections inside the P-time Event Graph. Relation $T_{l}^{-} \leq x_{p_{i}}(k)-x \cdot p_{l}\left(k-\mathbf{M}_{l}^{0}\right)$ of (1) for each place $p_{l} \in P$ is contained in the first rows of (2) relevant to $G^{-}$and $-T^{-}$while $x_{p_{l}}(k)-$ $x \bullet p_{l}\left(k-\mathbf{M}_{l}^{0}\right) \leq T_{l}^{+}$appears in the last rows relevant to $G^{+}$and $T^{+}$. Precisely, the following property defining $G^{-}$and $G^{+}$makes the connections with the rows of the usual incidence matrix $W$.

Property [3]

Let us assume that $m=1$. For each place $p_{l} \in P$, we have: $\left(G_{0}^{-}\right)_{l, .}=W_{l, \text {. }}$ and $\left(G_{1}^{-}\right)_{l, .}=0$ if $\mathbf{M}_{l}^{0}=0 ;\left(G_{0}^{-}\right)_{l, .}=-\left(W^{-}\right)_{l, .}$ and $\left(G_{1}^{-}\right)_{l, .}=\left(W^{+}\right)_{l, \text {. }}$ if $\mathbf{M}_{l}^{0}=1$. Moreover, $G^{+}=-G^{-}$. 


\section{Critical subsystems}

3.1 Preliminary results

Let $H=\left(\begin{array}{c}G_{1}^{-}+G_{0}^{-} \\ G_{1}^{+}+G_{0}^{+}\end{array}\right), N=\left(\begin{array}{c}G_{0}^{-} \cdot u \\ G_{0}^{+} \cdot u\end{array}\right)$ and $\theta=\left(\begin{array}{c}-T^{-} \\ T^{+}\end{array}\right)$. The dimensions of these matrices are respectively $(2 . q \times n),(2 . q \times 1)$ and $(2 . q \times 1)$. Let $u$ be a unitary vector with $|u|=|x(k)|=n$, that is, $u=(1 \ldots 1)^{t}$. The following definition describes a 1-periodic behavior of TIM (2).

Definition 1 TIM (2) follows a 1-periodic behavior when its trajectory satisfies equality $x(k+1)=\lambda . u+x(k)$ for $k \geq 0$ where $\lambda$ is the cycle time.

The introduction of $x(k+1)=\lambda \cdot u+x(k)$ in TIM (2) gives the equivalent form

$$
\left(\begin{array}{c}
\left(G_{1}^{-}+G_{0}^{-}\right) \\
\left(G_{1}^{+}+G_{0}^{+}\right)
\end{array}\right) \cdot x(k)+\left(\begin{array}{l}
G_{0}^{-} \\
G_{0}^{+}
\end{array}\right) \cdot \lambda \cdot u \leq\left(\begin{array}{r}
-T^{-} \\
T^{+}
\end{array}\right)
$$

or

$$
H . x(k) \leq \theta-N . \lambda \text { for } k \geq 0 \text {. }
$$

which is the starting point of the study: if a pair $(x(k), \lambda)$ satisfies $(4)$, then TIM (2) can follow the relevant 1-periodic trajectory.

An objective is to determine the minimum or maximum cycle time such that the TIM (2) follows a 1-periodic behavior starting from the finite starting point $x(0)$ (i.e. the first firing dates of the transitions when time Petri nets are considered) which is limited by the addition of the constraint $x(0) \geq L$ in the problem of minimization $(x(0) \leq L$ in the problem of maximization) with $L \in \mathbb{R}^{n}$. These constraints present a practical point of view as we can consider the example of a company where the working day starts at 8.00. A current rule is that each task $i$ must start after 8.00 as the company is closed before: we have $x_{i}(0) \geq L_{i}$ with $L_{i}=8.00$ for $i=1, \ldots, n$. A symmetrical rule is as follows: each task $i$ must start before 8.00 and we have $x_{i}(0) \leq L_{i}$.

Assuming the existence of a solution in (4), the problems of minimization and maximization of the cycle time $\lambda$ can be written as follows. The optimal cycle time such that the TIM (2) follows a 1-periodic behavior starting from a finite initial starting point $x(0)$ is the solution to the following linear programming problem.

\section{Problem I}

$L)$.

$\min (\lambda)$ (resp., $\max (\lambda))$ under constraint $(4)$ with $x(0) \geq L$ (resp., $x(0) \leq$

Deduced from a variant of the famous Farkas' Lemma (Corollary 7.1.e in [11]), the following theorem focuses on the existence of a pair $(x(0), \lambda)$ in (4) without the computation of a starting point $x(0)$ as in Problem I. Let $Y=\left\{y \in \mathbb{R}^{2 \cdot q} \mid y \cdot H=0\right.$ with $\left.y \geq 0\right\}$ with the following partition $Y=$ $Y^{-} \cup Y^{=} \cup Y^{+}$exploited below. 


$$
\begin{aligned}
& Y^{-}=\{y \in Y \mid y \cdot N=-1\} \\
& Y^{=}=\{y \in Y \mid y \cdot N=0\} \\
& Y^{+}=\{y \in Y \mid y \cdot N=1\}
\end{aligned}
$$

Theorem 1 [3] System (4) has a solution $(x(0), \lambda)$ if and only if the two following conditions are satisfied:

$$
\begin{gathered}
\left(\forall y \in Y^{=}\right) y \cdot \Theta \geq 0 \\
\lambda_{\min }=\max _{y \in Y^{-}}(-y \cdot \Theta) \leq \lambda_{\max }=\min _{y \in Y^{+}}(y \cdot \Theta)
\end{gathered}
$$

In other words, the two inequalities (6) and (7) express general conditions of existence of a pair $(x(0), \lambda)$. The first one is a constraint independent of the cycle time while the second one gives an interval of possible values $\left[\lambda_{\min }, \lambda_{\max }\right]$ which can be taken for the cycle time. In the case of a Timed Event Graph, $\lambda_{\min }$ is the value of the usual optimal cycle while $\lambda_{\max }$ is infinite. In fact, the previous theorem and Problem I implicitly consider a fixed event number $k$ which must be generalized to an infinite horizon: this generalization is made in Theorem 3 which exploits the following definition of homogeneity and Theorem 2 .

Definition 2 TIM (2) is said to be forward-homogeneous (resp., backwardhomogeneous) if any trajectory of TIM (2) is invariant by shifting with any positive delay (resp., negative delay), that is: if a trajectory $x(k)$ satisfies $(2)$, then the trajectory $x(k)+\omega \cdot \Delta . u$ with $\omega=1$ (resp., $\omega=-1$ ) also satisfies (2) for any delay $\Delta \in \mathbb{R}$ with $\Delta>0$. TIM (2) is said to be strictly-homogeneous when it is forward-homogeneous and backward-homogeneous.

The following theorem gives an algebraic way to analyze the homogeneity of the TIM (2).

Theorem 2 [4] TIM (2) is forward-homogeneous (resp., backwardhomogeneous) if and only if $H . \omega . u \leq 0$ with $\omega=1$ (resp., $\omega=-1$ ). TIM (2) is strictly-homogeneous if and only if $H . u=0$.

\subsection{Main objective}

Let us analyze the 2.q relations of TIM (2) where $I=\{1, \ldots, 2 . q\}$ is the set of the relevant indexes. For simplicity, we do not distinguish a relation and its relevant index and, a relation whose index is $i$ is directly written "relation $i$ ". The main objective of this paper is the determination of the min-critical (resp., max-critical) relations of the TIM (2). In Timed Event Graphs, each circuit where the ratio equals the maximum ratio is a critical circuit as a small variation of any time durations relevant to the corresponding critical places can modify the value of the minimum cycle time. In this paper, the concept of critical places relevant to the critical circuits is replaced by the notion of critical relations which is now defined. 
Definition 3 The relation

$$
\left(\begin{array}{l}
G^{-} \\
G^{+}
\end{array}\right)_{i, .} \cdot\left(\begin{array}{l}
x(k) \\
x(k+1)
\end{array}\right) \leq \Theta_{i},
$$

for $i \in I$ in TIM (2) is said min-critical (resp., max-critical) for the minimization (resp., maximization) if there is a small variation of $\Theta_{i}$ which modifies the optimal value of the cycle time.

Therefore, the goal is now to specify the connections between variations of a time duration and a degradation of an optimal cycle time, and to provide the relevant exact variation signs, when this connection exists.

\subsection{Approach}

Before presenting the proposition and the algorithm determining the critical relations, Theorem 1 is now extended to an infinite horizon. The addition of an algebraic condition allows to avoid the development of the relations (4) on an infinite horizon $k=0,1,2,3, \ldots$

Theorem 3 When H.w.u $\leq 0$ with $\omega=1$, TIM (2) satisfies $x(k+1)=$ $\lambda . u+x(k)$ for any $k \geq 1$ for some given cycle time $\lambda$, if and only if constraints (6) and (7) are satisfied.

\section{Proof.}

Firstly, Theorem 2 shows that if $H . \omega . u \leq 0$ with $\omega=1$ the TIM (2) is forward-homogeneous. By definition, if an elementary trajectory $\{x(k), x(k+$ $1)\}$ satisfies (2), we can deduce that $\{x(k)+\omega \cdot \Delta \cdot u, x(k+1)+\omega \cdot \Delta \cdot u\}$ also satisfies (2) for any $\Delta \in \mathbb{R}$. Particularly, we can take $\Delta=\lambda$ and if $\{x(0), x(1)=$ $x(0)+\lambda . u\}$ satisfies (2) which expresses the starting of the trajectory, then $\{x(1)=x(0)+\lambda \cdot u, x(2)=x(0)+2 \cdot \lambda \cdot u\}$ also satisfies (2) and the reasoning can be repeated for $\{x(k), x(k+1)\}$ with $k \geq 2$. Therefore, if H.w.u $\leq 0$ with $\omega=1$, the existence of the elementary trajectory $\{x(0), x(1)=x(0)+$ $\lambda . u\}$ satisfying (2) implies that the TIM (2) can follow a relevant 1-periodic behavior on an infinite horizon starting from $x(0)$. Secondly, the existence of the elementary trajectory $\{x(0), x(1)=x(0)+\lambda . u\}$ satisfying $(2)$ is equivalent to the satisfaction of (4) which has been obtained by elementary manipulations. Finally, the application of Theorem 1 gives the equivalence of the existence of a solution in (4) and the satisfaction of the two constraints (6) and (7).

As theorem 3 allows a complete analysis on an infinite horizon, relations (7) can be exploited as each non-null component of vector $y$ highlights a relation of TIM (2) which corresponds to a min-critical relation if $y$ satisfies $y . \Theta=\lambda_{\min }$ (resp., max-critical relation if $y$ satisfies $y . \Theta=\lambda_{\max }$ ). The following result gives an efficient way of determining the critical relations and specifies the suited sign of the variation of the time duration in definition 3 . Note that the consideration of a vector $y \in Y^{-}$which is not relevant to the minimum, that is, $-y . \Theta<\lambda_{\min }$ (resp., maximum, that is, $\lambda_{\max }<y . \Theta$ ) cannot produce a 
variation of the optimal solution for a small variation of the time durations contained in $\Theta$. The two cases in following proposition consider the same variation of the entry $\Theta_{i}$ that is, a decrease.

Proposition 1. Assuming that the constraints $H \cdot \omega \cdot u \leq 0$ with $\omega=1$ and (6) are satisfied, relation $i \in\{1, \ldots, 2 . q\}$ in TIM (2) is:

- min-critical if any decrease of the entry $\Theta_{i}$ implies an increase of $\lambda_{\min }$.

- max-critical if any decrease of the entry $\Theta_{i}$ implies a decrease of $\lambda_{\max }$.

\section{Proof.}

Below, the decrease of the entry $\Theta_{i}$ is denoted by the positive value $\Delta_{i}$ : formally, $\Delta_{i}>0$. Applying theorem 3 , we can now analyze the remaining constraint (7). Let us consider a row-vector $y \in Y^{-}$leading to the optimal solution $\lambda_{\min }$ : So, $-y \cdot \Theta=\lambda_{\min }$ otherwise $-y \cdot \Theta<\lambda_{\min }$. Firstly, for any modification of $\Theta$, vector $y$ is still a solution of the constraints as the sets $Y$ and $Y^{-}$do not depend on the time durations. The expression $-y \cdot \Theta=\lambda_{\min }$ shows that the decrease of $\Theta_{i}$ implies an increase of $\lambda_{\min }$ for $y_{i} \neq 0$ as the relevant optimal solution $\lambda_{\min }^{\prime}$ is the maximum of the different products. We obtain $\lambda_{\min }^{\prime}=\lambda_{\min }+y_{i} \cdot \Delta_{i}$. Precisely, as $\Theta=\left(\left(-T^{-}\right)^{t}\left(T^{+}\right)^{t}\right)^{t}$, a decrease of a component $i$ of $\Theta$ corresponds to an increase of an entry $i$ of $T^{-}$or a decrease of an entry $i$ of $T^{+}$. The modified problem gives a greater optimal solution $\lambda_{\min }^{\prime}=\lambda_{\min }+y_{i} . \Delta_{i}$ for a decrease of an entry $i$ of $T^{+}\left(T_{i}^{+}-\Delta_{i}\right)$ or for an increase of an entry $i$ of $T^{-}\left(T_{i}^{-}+\Delta_{i}\right)$. The value of coefficient $y_{i}$. shows the sensitivity to a variation of the time parameter $T_{i}^{-}$or $T_{i}^{+}$. For $\lambda_{\max }=$ $y . \Theta$, the reasoning is similar. For $y \in Y^{+}$and a decrease of an entry of $\Theta$, the modified problem gives a lower optimal solution $\lambda_{\max }^{\prime}=\lambda_{\max }-y_{i} . \Delta_{i}$.

As the previous result shows the effects on the optimal solution $\lambda_{\min }$ or $\lambda_{\max }$ which restrict the interval $\left[\lambda_{\min }, \lambda_{\max }\right]$, the determination of the critical parts can be made by the following algorithm based on the checking of each time duration.

Algorithm of detection of min-critical and max-critical relations. Let $\Delta$ be an arbitrary small positive scalar $(\Delta>0)$

For each relation $i \in I$ in TIM (2) $\Theta_{i} \leftarrow \Theta_{i}-\Delta$ (that is, $T_{i}^{-} \leftarrow T_{i}^{-}+\Delta$ or $T_{i}^{+} \leftarrow T_{i}^{+}-\Delta$ resp.)

Compute the optimal solution $\lambda_{\min }^{\prime}$ and $\lambda_{\max }^{\prime}$ by any technique If $\lambda_{\min }^{\prime}>\lambda_{\min }$, then the relation $i$ (the pair $\left(p_{i}, T_{i}^{-}\right)$or $\left(p_{i}, T_{i}^{+}\right)$resp.) is min-critical

\section{is max-critical}

$$
\text { If } \lambda_{\max }^{\prime}<\lambda_{\max } \text {, then the relation } i \text { (the pair }\left(p_{i}, T_{i}^{-}\right) \text {or }\left(p_{i}, T_{i}^{+}\right) \text {resp.) }
$$

\section{End-for}

Based on the computation of the cycle time which can be made by any technique, the approach generates values of the cycle time inside the interval $\left[\lambda_{\min }, \lambda_{\max }\right]$. It can be seen as a slight restriction of the evolution as the modification of the time duration has led to slow down or to accelerate the process described by the TIM (2) respectively. 


\subsection{Practical case study}

Represented by a P-time Event Graph in Fig. 1, this example considers a baking process in a plant bakery composed of two processes giving a bread of higher quality (upper range 1) and of an ordinary quality (ordinary range 2) respectively. The two ranges follow the same production sequence on the four machines which are the kneading machine, the dividing machine, the molding machine and the oven: they are described by places $p_{1}, p_{2}, p_{3}, p_{4}, p_{5}, p_{6}, p_{19}$ and $p_{7}, p_{8}, p_{9}, p_{10}, p_{11}, p_{12}, p_{13}, p_{18}$, respectively. The connections between the two production lines are expressed by places $p_{14}, p_{15}, p_{16}, p_{17}$. Taken from a semi-industrial plant bakery [6], the time durations specific for each range and an additional operation of proofing (place $p_{8}$ ) for the range 2 lead to two different qualities. The main operations are as follows. After mixing the ingredients, the dough is kneaded. This step of kneading is represented by places $p_{1}$ and $p_{7}$. This operation lasts between 15 and 27 minutes. The following step is the first proofing which is the process of leaving the dough in the machine for 10 to 30 minutes at a steady temperature of $27^{\circ} \mathrm{C}$ which leads to a fermenting. Only the process 2 contains this step (place $p_{8}$ ). Then, the dough is divided into pieces of about 900 grams each and placed in nets in the proofer (this step of dividing is represented by $p_{2}$ for the process 1 and $p_{9}$ for the process 2). The following step is the second proofing which lasts 180 minutes for the range $1\left(p_{3}\right)$ and 15 to 20 minutes for the range $2\left(p_{10}\right)$. Then each piece of dough is shaped with a molding machine (the molding step is represented by $p_{4}$ for the range 1 and $p_{11}$ for the range 2 ). Then the operation of final proofing provides the last fermenting ( $p_{5}$ for the range 1 and $p_{12}$ for the range 2). Finally, Baking the bread takes up 20 to 25 minutes at a temperature of 250 to $280^{\circ} \mathrm{C}$ in the oven and is identical for the two ranges (the baking corresponds to $p_{6}$ for the range 1 and $p_{13}$ for the range 2 ). The following operations as the cooling, the packing and the transport are not considered. Two dividing machines and two molding machines are available. We assume an upper limit of 300 minutes in the period of non-use of the dividing machines and molding machines otherwise another production line not described in the event graph can use these machines (places $p_{14}$ to $p_{17}$ ). The oven is assumed to be sufficiently large.

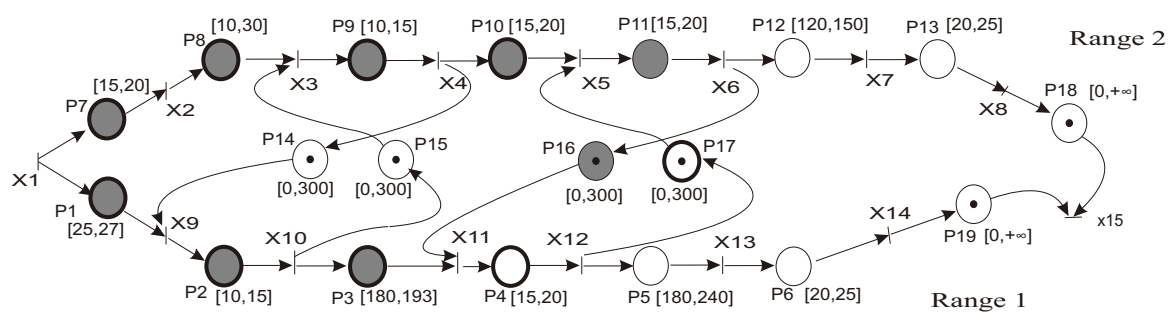

Fig. 1 Plant bakery 
The system contains 19 places and 15 transitions. By lack of place, the initial marking, the vectors and the matrices of the time interval model are not given but can directly be deduced from the figure.

The values of the minimum cycle time and maximum cycle time which are 145 and 190 respectively, are computed by applying the linear programming problems I with the function linpro() of the software Scilab. The CPU time of the execution of a linear programming problem is around 0.011 s with a PC Intel Core $2.93 \mathrm{GHz}$ and $0.9 \mathrm{~s}$ for the 78 problems of the complete approach.

For $\lambda_{\min }=145$, the computation of the 38 problems based on a decrease of the component $\Theta_{i}$ with $\Delta=0.1$ (that is, an increase of $T_{i}^{-}$or a decrease of $T_{i}^{+}$resp.) leads to an increase of the cycle time where $\lambda=145.1$ for 9 problems. Represented by the bold circle of the places in Fig. 1, the relevant set of min-critical pairs is

$P_{\text {min }}^{\text {crit }}=\left\{\left(p_{1}, T_{1}^{-}\right),\left(p_{2}, T_{2}^{-}\right),\left(p_{3}, T_{3}^{-}\right),\left(p_{4}, T_{4}^{-}\right),\left(p_{17}, T_{17}^{-}\right),\left(p_{7}, T_{7}^{+}\right),\left(p_{8}, T_{8}^{+}\right)\right.$, $\left.\left(p_{9}, T_{9}^{+}\right),\left(p_{10}, T_{10}^{+}\right)\right\}$while the remaining pairs yield the cycle time value $\lambda=$ $\lambda_{\min }=145$ despite the variation of the duration. For $\lambda_{\max }=190$, the computation of the 38 problems based on the variation of each time duration with $\Delta=0.1$ leads to a variations of the cycle time where $\lambda=189.9$ for 9 problems. Represented by the grey places in Fig. 1, the relevant set of max-critical pairs is

$P_{\max }^{c r i t}=\left\{\left(p_{1}, T_{1}^{-}\right),\left(p_{2}, T_{2}^{-}\right),\left(p_{3}, T_{3}^{-}\right),\left(p_{7}, T_{7}^{+}\right),\left(p_{8}, T_{8}^{+}\right),\left(p_{9}, T_{9}^{+}\right),\left(p_{10}, T_{10}^{+}\right)\right.$, $\left.\left(p_{11}, T_{11}^{+}\right),\left(p_{16}, T_{16}^{+}\right)\right\}$while the remaining pairs yield the cycle time value $\lambda=$ $\lambda_{\max }=190$ despite the variation of the duration. So, the set $P_{\mathrm{min}}^{c r i t}$ as well as $P_{\max }^{c r i t}$ depends on the lower and upper bounds of the time durations and does not correspond to some (oriented) circuits of the event graph.

Let us illustrate a practical interest in the optimization of the process. We can desire to keep the best cycle time $\lambda_{\min }$ which corresponds to the greatest production rate and so, to avoid a general slow-down of the whole process. It implies that a greater time duration of the kneading is not possible for the range 1 (as $\left(p_{1}, T_{1}^{-}\right)$is critical) but possible for the range 2 (as $\left(p_{7}, T_{7}^{-}\right)$ is not critical). So, the choice of a reliable machine is necessary in the first case. The remark is similar for the two dividing steps (as $\left(p_{2}, T_{2}^{-}\right)$is critical but not $\left.\left(p_{9}, T_{9}^{-}\right)\right)$and the molding steps (as $\left(p_{4}, T_{4}^{-}\right)$is critical but not $\left.\left(p_{11}, T_{11}^{-}\right)\right)$. Therefore, if we consider the kneading, the dividing and the molding for range 2 which are not relevant to critical pairs, an energy saving and so a financial economy can be obtained by reducing the speed of the relevant machines without modifying the best production rate of the entire process. Another possibility is the replacement of the relevant machines of the range 2 by less efficient machines. Therefore, we can make an adaptation of the algorithm of detection of the critical relations which computes the greatest $\Delta$ with the condition that the optimal cycle time $\lambda_{\min }$ is kept and the system remains consistent. For $\lambda_{\min }$, the results are as follows: the new values of time durations for $T_{7}^{-}, T_{9}^{-}$and $T_{11}^{-}$can be 20,15,20 while the initial values are 15 , 10,20 , respectively. The same technique can be made if we desire to make an optimization of the machine speeds for the maximum cycle time $\lambda_{\max }$. 


\section{Conclusion}

Contrary to the time-costly enumerative technique extracting every circuit, the proposed approach considers each time duration. Exploiting the variations of durations, the approach produces the min-critical and max-critical subsystems where each critical subsystem depends on the lower and upper bounds of the time durations together. The technique is effective as it only needs the application of polynomial algorithms of linear programming [11]. Showing that the graphical concepts must be surpassed, the practical example illustrates that the critical subsystems are not limited to the classical critical circuits of the event graph. As suggested by the practical case study in Section 3.4, a natural perspective is the cost optimization of the resources and machines without reducing the production rate and the service quality of the process.

\section{References}

1. F. Baccelli, G. Cohen, G.J. Olsder and J.P. Quadrat. Synchronization and Linearity. An Algebra for Discrete Event Systems, available from http://maxplus.org, New York, Wiley, 1992.

2. S. Bernardi and J. Campos. A min-max problem for the computation of the cycle time lower bound in interval-based Time Petri Nets, IEEE Transactions on Systems, Man, and Cybernetics: Systems, Vol.: 43, Issue: 5, Sept. 2013.

3. P. Declerck, "Cycle Time of a P-time Event Graph with Affine-Interdependent Residence Durations," Journal of Discrete Event Dynamic Systems, November 2013, available on http://perso-laris.univ-angers.fr $/ \sim$ declerck/.

4. P. Declerck, "Extremum Cycle Times in Time Interval Models," IEEE Trans. Autom. Control, Vol. 63, Issue 6, pp. 1821-1827, June 2018, available on http://perso-laris.univangers.fr/ declerck/.

5. A. Giua, A. Piccaluga and C. Seatzu, "Optimal Token Allocation in Timed Cyclic Event-Graphs," Proc. 4th Workshop on Discrete Event Systems, pp. 209-218, August 2000 .

6. C. Gros, DEA report in "Biotechnologies et Industries Alimentaires", Ecole Nationale Supérieure d'Agronomie et des Industries Alimentaires (ENSAIA), INPL, 2000.

7. T.-E. Lee, S.-H. Park, and C. Jung, "Steady State Analysis of Timed Event Graph with Time Window Constraints," Discrete Applied Mathematics, Vol. 167, pp. 202-216, 2014.

8. C. Hanen and A. Munier Kordon, "Periodic schedules for linear precedence constraints," Discrete Applied Mathematics, Vol. 157, Issue 2, pp 280-291, Jan. 2009.

9. T. Murata. Petri Nets: Properties, Analysis and Applications, Proceedings of the IEEE, Vol. 77, No. 4, 1989.

10. Rodríguez, Ricardo J. \& Júlvez, Jorge. (2010). Accurate Performance Estimation for Stochastic Marked Graphs by Bottleneck Regrowing. From book Computer Performance Engineering - 7th European Performance Engineering Workshop, EPEW 2010, Bertinoro, Italy, September 23-24, 2010. Proceedings (pp.175-190)

11. A. Schrijver. Theory of linear and integer programming, John Wiley and Sons, 1987. 